\title{
Une innovation incrémentielle : la conception et la diffusion d'un pivot d'irrigation artisanal dans le Souf (Sahara algérien).
}

\author{
Abdelkrim Ould Rebai ${ }^{1, *}$, Tarik Hartani ${ }^{1,2}$, Mohamed Nacer Chabaca ${ }^{1}$ et Marcel Kuper ${ }^{3,4}$ \\ ${ }^{1}$ École nationale supérieure agronomique (ENSA), Rue Hassen Badi Belfort, El Harrach, 1600 Alger, Algérie \\ 2 Centre universitaire de Tipaza, Ouade Merzouk, 42000 Tipaza, Algérie \\ 3 Cirad UMR G-eau TA C-90/15, 73, rue Jean-Francois Breton, 34398 Montpellier cedex 5, France \\ ${ }^{4}$ Institut agronomique et vétérinaire (IAV Hassan II), 10100 Rabat, Maroc
}

\begin{abstract}
Résumé - On observe depuis une vingtaine d'années l'émergence de bassins de production maraîchère dans le Sahara algérien par la mobilisation des eaux souterraines profondes, en marge des oasis traditionnelles. Dans la vallée du Souf, les agriculteurs se sont orientés vers la culture de la pomme de terre, irriguée par des petits pivots artisanaux. L'objectif de l'article est d'analyser le processus d'innovation ayant permis l'émergence et la diffusion du pivot artisanal. Nous avons caractérisé le dispositif technique des différents pivots conçus successivement et mené des entretiens semi-directifs auprès de 24 agriculteurs, dix artisans, quatre commerçants locaux et quatre conseillers de sociétés d'agrofourniture. La conception et la mise en place du pivot artisanal résultent d'une innovation incrémentielle à partir des grands pivots conventionnels introduits par l'État, qui étaient coûteux et non adaptés aux objectifs et aux capacités techniques des agriculteurs. De là vient la production et la diffusion par des artisans locaux d'un système d'irrigation accessible et adapté aux réalités socio-économiques de la région. L'association entre pivot artisanal et pomme de terre a renforcé la dynamique agricole dans le Souf avec la production annuelle de plus de 1 million de tonnes de pomme de terre grâce à 35000 pivots artisanaux, soit $35 \%$ de la production nationale. Cependant, ce système de production dépend de l'utilisation des eaux souterraines peu renouvelables et est positionné sur des marchés agricoles volatiles, ce qui nous amène à interroger sa durabilité.
\end{abstract}

Mots clés : irrigation / pivot artisanal / innovation incrémentielle / Oued Souf / Sahara / Algérie

\begin{abstract}
An incremental innovation: design and diffusion of an artisanal center pivot for irrigation in the Souf (Algerian Sahara). In the Algerian Sahara, several horticultural production areas emerged on the margins of traditional oases over the past twenty years through the use of deep groundwater. In the Souf valley, farmers produce potatoes irrigated by small artisanal center pivots. The aim of the article is to analyze the innovation process that led to the design and diffusion of artisanal center pivots by local craftsmen. An analysis was made of the material composition of the different center pivots that were implemented consecutively, followed by semi-structured interviews with 24 farmers and with 10 local craftsman, four skilled workmen and traders. The design and the diffusion of these pivots came about through an incremental innovation process that started by deconstructing the large-scale conventional pivots introduced by the State, which were costly and unsuited to the ambitions and technical capacities of farmers. This resulted in a flexible system adapted to the local socio-economic realities. The association of local pivots and potato farming contributed to the agricultural dynamics in the Souf with the annual production of over 1 million tons of potatoes in more than $35000 \mathrm{ha}$, which represents $35 \%$ of the national production. Nevertheless, this farming system depends on the use of little renewable groundwater and on volatile agricultural markets, which questions its sustainability.
\end{abstract}

Keywords: irrigation / pivot / incremental innovation / Oued Souf / Sahara / Algeria

\footnotetext{
* Auteur de correspondance : ouldrebaikarim@yahoo.fr
} 


\section{Introduction}

Dans le Sahara algérien, l'émergence de bassins de production de cultures maraîchères primeurs a été rendue possible par des conditions climatiques favorables, des ressources suffisantes en terres et en eaux souterraines, une importante main-d'œuvre agricole et une demande croissante des marchés nationaux (Dubost et Larbi, 1998). À côté du palmier dattier (et notamment la variété commerciale Deglet nour) en pleine expansion, et en marge des oasis traditionnelles, la tomate et le poivron sous serre à Biskra, le melon et la pastèque à Ouargla, la tomate à Adrar, sont devenus des cultures emblématiques du Sahara depuis une vingtaine d'années. Le département d'El Oued est ainsi devenu le premier producteur de pomme de terre dans le pays et a produit 1,1 million de tonnes de pomme de terre en 2014 , soit $35 \%$ de la production nationale pour un montant estimé à 360 millions $€$.

Le développement horticole d'El Oued s'est opéré dans un contexte de déclin du système hydraulique traditionnel appelé Ghout, propre à la région. Les Ghouts sont des cuvettes artificielles creusées par l'homme, dans lesquelles les palmiers sont plantés par groupe de 20 à 100 et s'alimentent en eau dans la nappe phréatique. Le développement du pompage privé et étatique dans les nappes profondes, pour répondre aux besoins en eau potable de la population, a conduit, en l'absence d'un système de drainage, à une suralimentation de la nappe phréatique. Dès 1980, une remontée progressive et persistante de la nappe phréatique a été observée à El Oued et les premiers palmiers sont morts par asphyxie. En 2004, 231540 palmiers, sur un total de 742525 (soit $31 \%$ ), étaient morts asphyxiés dans la vallée du Souf (Côte, 2006).

L'émergence et l'extension de la pomme de terre dans la région d'El Oued sont étroitement associées à la diffusion de la technique de l'irrigation par pivot. Cette technique, originaire des États-Unis d'Amérique, a été développée au Moyen Orient et en Afrique du Nord par des grands programmes étatiques, à partir des années 1970/1980 (Bisson, 2003). L'ambition était d'impulser une agriculture qui se voulait moderne, en particulier la céréaliculture pour couvrir les besoins alimentaires de ces pays. Cependant, la diffusion de ces grands pivots a rencontré de nombreux échecs, y compris en Algérie, pour des raisons techniques, agronomiques et socio-économiques (Otmane et Kouzmine, 2013). Ainsi, au cours des années 1980, la région d'Oued Souf a été concernée par un grand programme étatique céréalier sous pivots. Ces pivots avaient une longueur de $350-400 \mathrm{~m}$ et pouvaient irriguer des superficies de 39 à 50 ha. La technologie était coûteuse et non adaptée aux objectifs et aux capacités techniques des agriculteurs. Cependant, dans les années 1990, ce programme a inspiré des artisans locaux pour concevoir un pivot artisanal de taille plus modeste, avec une longueur de 25 à $50 \mathrm{~m}$, pour irriguer des cultures maraîchères sur des parcelles de 0,5 à 1 ha, en particulier la pomme de terre. Il s'en est suivi une extension rapide de la superficie cultivée en pomme de terre dans cette région. L'objectif de l'article est d'analyser le processus d'innovation incrémentielle ayant permis l'adaptation du modèle du pivot importé. Cette analyse permet de saisir les raisons de l'attractivité du pivot artisanal utilisé aujourd'hui sur des milliers d'hectares pour l'irrigation de la pomme de terre, la deuxième culture stratégique du pays après les céréales.
Nous faisons l'hypothèse que le succès du pivot artisanal dans le Souf tire son origine de sa capacité à susciter l'adhésion de nombreux alliés de l'innovation (utilisateurs, fabricants, intermédiaires...) à travers un réseau socioprofessionnel dense (Akrich et al., 1988). L'analyse par les systèmes d'innovation permet de comprendre comment une multitude d'acteurs interagissent, échangent des informations et des connaissances, et développent, diffusent et utilisent les innovations (Edquist, 2005 ; Ortiz et al., 2013). L'adoption des innovations ne peut avoir lieu sans réunir les facteurs susceptibles d'enrôler les acteurs par la création d'un environnement sociotechnique approprié (Akrich et al., 1988; Benouniche et al., 2014). Pour le secteur de l'agriculture, de nombreux auteurs ont montré que les acteurs conventionnels, tels que les services de l'agriculture et de la vulgarisation, ne sont pas les seuls acteurs de l'innovation (Benouniche et al., 2016). Des agriculteurs, des intermédiaires, des artisans et des commerçants, qu'ils agissent de façon individuelle ou collective, peuvent contribuer à réécrire « la grammaire de l'innovation », et à faire évoluer les connaissances et même les objectifs des processus d'innovation (Knickel et al., 2009).

\section{Zone d'étude et méthodologie}

La région d'Oued Souf fait partie du département d'El Oued situé dans le Sud-Est algérien (Fig. 1). Elle relève du Sahara septentrional et du Grand Erg oriental, couvert par une chaîne de dunes sableuses. Elle se caractérise par un climat hyper-aride, une température moyenne annuelle de l'ordre de $22^{\circ} \mathrm{C}$ et des précipitations moyennes annuelles de $78 \mathrm{~mm}$ (ONM, 2012). La région dispose d'une réserve hydrique souterraine importante constituée de trois aquifères: un aquifère libre et deux aquifères captifs, le complexe terminal et le continental intercalaire.

La région connaît depuis 2000 une importante dynamique agricole illustrée par le développement de la filière pomme de terre. Cette culture est irriguée par des pivots «artisanaux», conçus et fabriqués localement, pour une surface moyenne irriguée d'un hectare. Les superficies concernées sont passées de 800 ha en 2000 à 35000 ha en 2013, ce qui représente environ 35000 pivots installés en moins de 15 ans, selon les statistiques du ministère de l'Agriculture.

Trois communes ont été retenues pour cette étude, sur la base des données collectées auprès des services locaux et d'entretiens semi-directifs réalisés avec quatre cadres administratifs :

Les communes de Guemar et Reguiba constituent des territoires où les premiers pivots artisanaux ont été fabriqués et adaptés progressivement aux réalités socio-économiques de la région.

La commune de Hassi Khalifa est actuellement leader de la filière pomme de terre sous pivots; environ $25 \%$ de la production du département d'El Oued y est concentrée. Les superficies emblavées dans la commune sont passées de 125 ha en 2004 à 8000 ha en 2013, pour une production totale de 269360 tonnes (sur un total de 1172500 tonnes pour l'ensemble du département).

Des entretiens avec quatre conseillers salariés des sociétés d'agrofournitures nous ont permis de repérer les sites de production de la pomme de terre et les pivots artisanaux à 


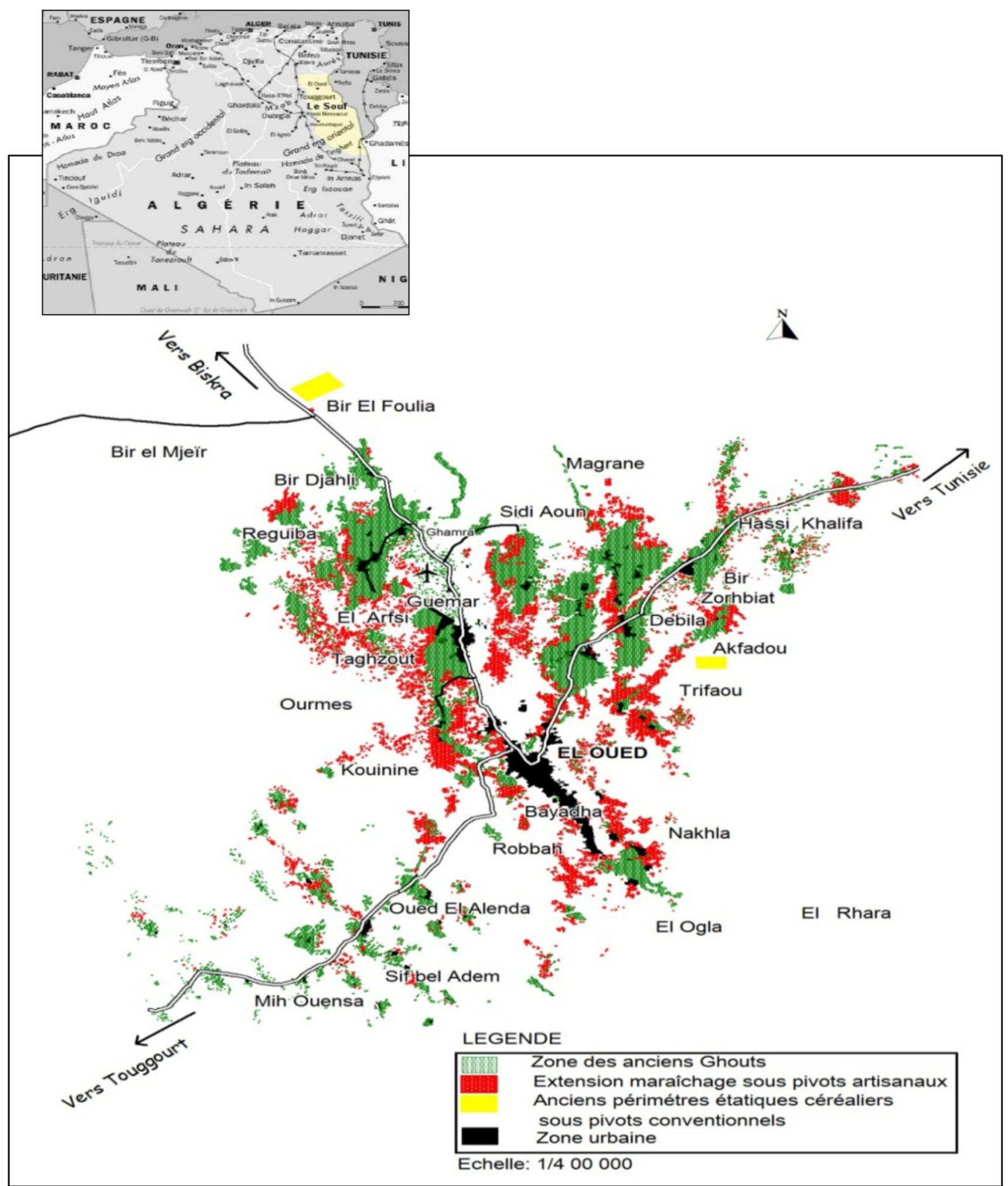

Fig. 1. Présentation de la vallée d'Oued Souf : localisation et occupation du sol (adapté à partir de la carte d'occupation des sols INSID, 2011). Fig. 1. Presentation of Oued Souf Valley: location and occupation of sol (adapted from the occupation map INSID, 2011).

investiguer. Nous avons mené des enquêtes auprès de 24 griculteurs utilisant des pivots artisanaux dans la commune de Hassi Khalifa. Ces exploitations ont été choisies parmi les utilisateurs des pivots artisanaux et ce, selon trois catégories
- grands investisseurs, petits investisseurs et fellahs (cultivateurs) - pour représenter les différentes stratégies d'utilisation du pivot artisanal, notamment le nombre de pivots installés et les systèmes de culture pratiqués (Tab. 2). Les 
observations effectuées dans ces 24 exploitations ont permis de décortiquer les différents composants du pivot artisanal et de suivre les flux de ces composants, tout en recensant les différents acteurs impliqués. À cela s'ajoutent des entretiens semi-directifs auprès de dix artisans locaux dans les deux communes de Guemar et Reguiba, qui constituent encore aujourd'hui un centre artisanal de fabrication très important dans la zone. Ces artisans sont impliqués dans l'approvisionnement en pièces détachées ou en composants de base, la fabrication des composants, l'assemblage et le montage des pivots artisanaux. Enfin, nous avons réalisé des entretiens auprès de quatre commerçants chargés de la distribution et des ventes des équipements et des pièces importées composant les pivots artisanaux.

\section{Résultats}

\subsection{Co-conception du pivot artisanal : un processus d'innovation à partir des pivots conventionnels}

Vers la fin des années 1980 et avec le déclin des Ghouts, les agriculteurs du Souf étaient à la recherche de systèmes de production alternatifs. Ils se sont orientés progressivement vers les cultures maraîchères et industrielles, principalement les arachides irriguées à la raie en mobilisant un réseau de seguias (rigoles d'amenée) revêtues de plâtre pour éviter les pertes en eau. Cependant, les agriculteurs rencontraient des difficultés dans la réalisation et l'entretien des seguias, car cela nécessitait beaucoup de main-d'œuvre, non seulement pour la construction du réseau de seguia et leur revêtement, mais aussi pour l'évacuation continue du sable hors des rigoles. Ces difficultés ont motivé le fils d'un fellah de Ghamra (dans la commune de Guemar), un jeune lycéen de 21 ans passionné d'électromécanique, à envisager des alternatives. Inspiré par un pivot conventionnel du programme étatique céréalier se trouvant au village Bir El Foulia (commune de Reguiba) et qui était en panne, ce jeune s'est intéressé à la conception d'un pivot de plus petite taille. En 1994, il a procédé à une prospection auprès des ferrailleurs et des artisans locaux pour trouver les compétences nécessaires, notamment un soudeur et un tourneur, deux artisans expérimentés de son voisinage qui sont devenus ses associés. Ils ont établi une liste des pièces et matériaux nécessaires et la réalisation a été entamée dès leur acquisition. Six mois plus tard, le premier modèle de pivot (le modèle asymétrique à minuterie; Tab. 1) était entièrement fabriqué par cet innovateur. Celui-ci a alors commencé à recevoir des commandes de ses voisins agriculteurs. Au début de 1996, les premiers pivots ont été vendus à des agriculteurs de Ghamra. Les agriculteurs des communes de Guemar et Reguiba, après avoir observé et apprécié ce premier modèle de pivot artisanal, ont commencé à se rapprocher des artisans locaux (soudeurs, tourneurs et mécaniciens) pour les inciter à en fabriquer d'autres selon les mêmes principes.

«Pour ces agriculteurs, une solution miracle venait d'apparaître pour investir dans le maraîchage après la crise des Ghouts due à la remontée des nappes. D'un côté, il mettait fin aux difficultés d'entretien de la seguia et permettait de réduire les coûts de main-d'œuvre. De l'autre côté, ce pivot artisanal n'exigeait pas d'être démonté à chaque fin de campagne d'irrigation contrairement au système d'aspersion classique que l'État voulait développer dans le Souf» (un fellah de Ghamra, 60 ans, 8 ha).

À leur tour, des soudeurs des deux communes de Guemar et Reguiba ont commencé à apporter des adaptations au pivot. Selon l'innovateur, la mise au point du premier modèle commercialisé, asymétrique à minuterie, a permis le partage de l'idée et a ouvert la voie à d'autres artisans pour éliminer les multiples inconvénients qu'il présentait (Tab. 1), notamment :

- des pannes fréquentes du réducteur de vitesse; à chaque fois il fallait remplacer certaines pièces des pivots installés, en particulier les volants de moteurs, pièces peu disponibles;

- des difficultés liées à la fixation de la rampe au moyen de fils en acier;

- des problèmes d'enroulement du câble électrique sur la rampe ainsi que sur l'ensemble du pivot; à chaque irrigation il fallait le dérouler.

En 1996, un deuxième concepteur, dans la commune de Guemar (soudeur, 45 ans), ayant bénéficié d'une formation mécanique en Allemagne, a présenté un nouveau modèle de pivot symétrique avec deux rampes de $25 \mathrm{~m}$ chacune, appelé «modèle éventail» (Fig. 2; Tab. 1). Ce nouveau modèle fonctionne et se déplace par pression hydraulique. Son alimentation en eau se fait par connexion directe sur le puits à l'aide d'une pompe qui propulse l'eau dans les deux rampes et assure la rotation. Les premiers fabricants, qui étaient les concepteurs pionniers de Guemar et Reguiba, ont commercialisé ce modèle tout en essayant d'améliorer le modèle asymétrique de Ghamra en levant les contraintes citées précédemment (Tab. 1). C'est ainsi que le pivot artisanal a connu une adaptation continue et une amélioration progressive, avant d'être plus largement diffusé et commercialisé, depuis l'an 2000, sous sa forme actuelle (Fig. 3).

\subsection{Fabrication du pivot artisanal : une innovation incrémentielle grâce à un réseau dense et organisé d'alliés}

La fabrication et la diffusion des pivots artisanaux sont subordonnées à une diversité d'acteurs dont l'enchaînement des interventions constitue un réseau dense de prestations de services et de fournitures (Fig. 4). Nous avons identifié trois étapes dans la fabrication et le montage des pivots:

- acquisition des pièces par l'importation ou la récupération de pièces détachées sur des équipements obsolètes;

- fabrication de certains composants dans les ateliers locaux;

- montage du pivot sur l'exploitation agricole et service après-vente (Fig. 4).

Des commerçants privés importateurs alimentent les grossistes du marché d'El Oued en composants industriels venant de Chine: organes d'arrosage, tubes galvanisés, moteurs électriques et réducteurs de vitesse. Ces équipements sont ensuite distribués au détail par des quincaillers. Les épaves de voitures constituent la deuxième source de composants pour les fabricants des pivots. Il s'agit de l'ensemble des composants assurant le déplacement des pivots, notamment les roues et leurs pièces porteuses ainsi que 
Tableau 1. Modèles de pivots artisanaux développés par l'innovation incrémentielle.

Table 1. Models of artisanal center pivots developed by incremental innovation.

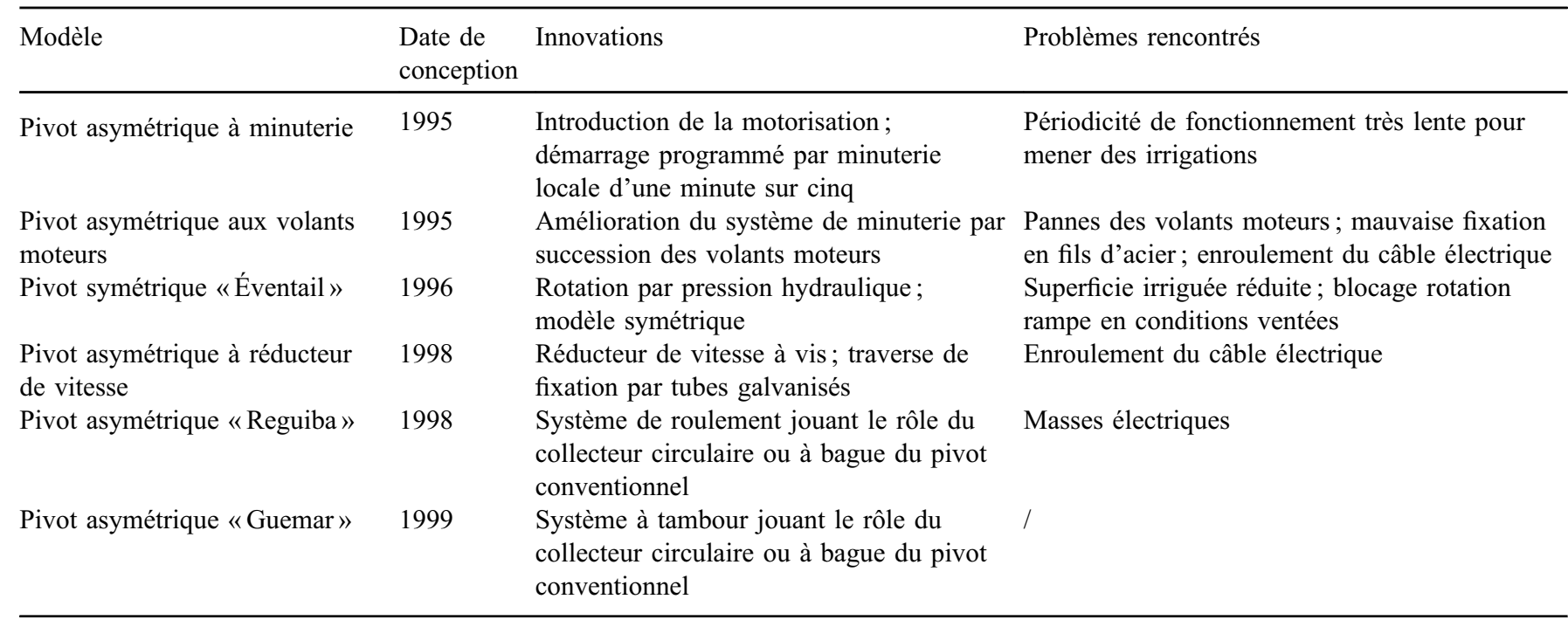
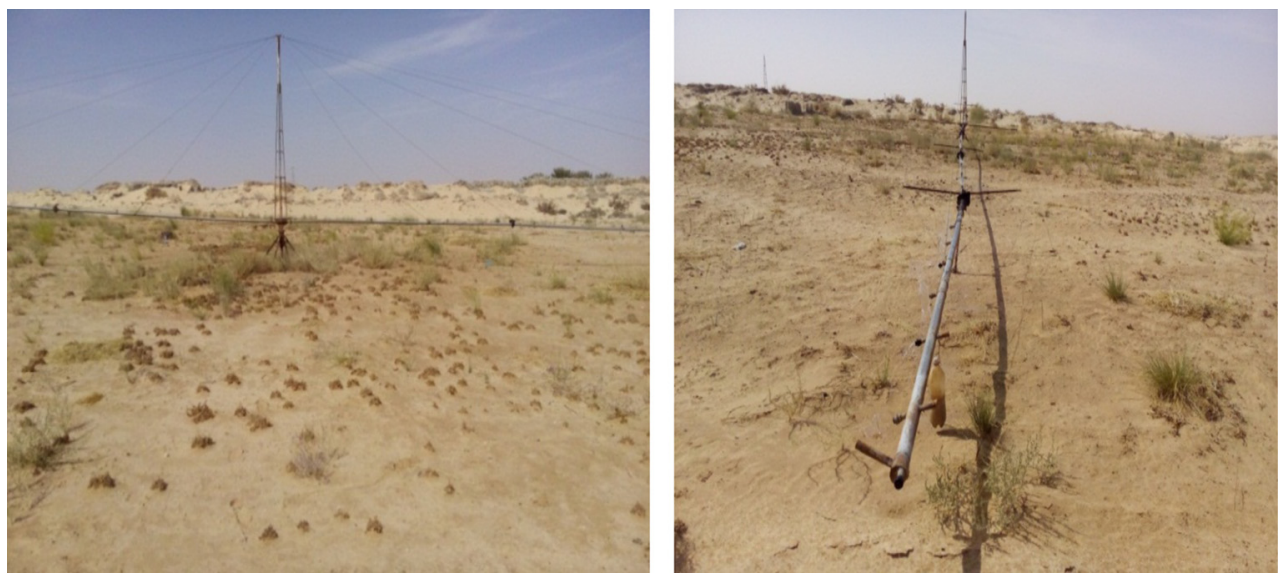

Fig. 2. Modèle du pivot symétrique «éventail».

Fig. 2. Model symmetrical pivot "fan".
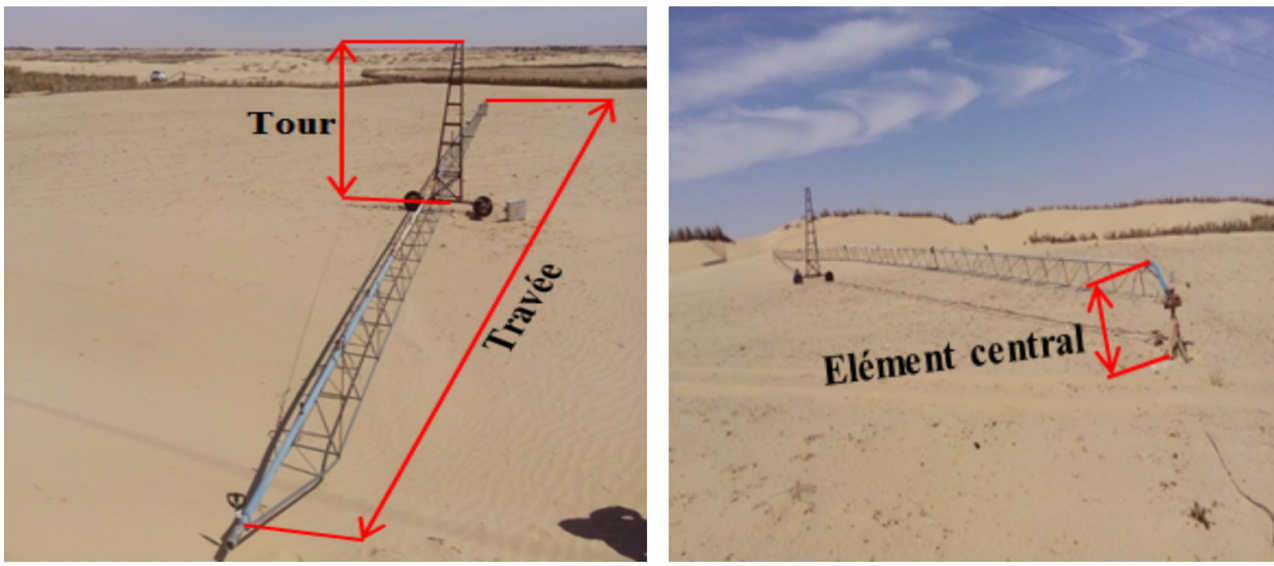

Fig. 3. Composantes du pivot artisanal. Fig. 3. Constituent of craft-trade pivot.

les roulements. Ces pièces sont achetées dans les casses automobiles et les garages. Ensuite, les artisans fabriquent dans leurs ateliers les composants qu'il est impossible d'acheter dans les filières précédentes. Les tourneurs produisent ainsi un modèle local de réducteurs de vitesse, un «réducteur à vis», qui est plus utilisé que le modèle industriel importé de Chine, en raison de son faible coût et de sa grande disponibilité dans le Souf. Ils produisent aussi des 


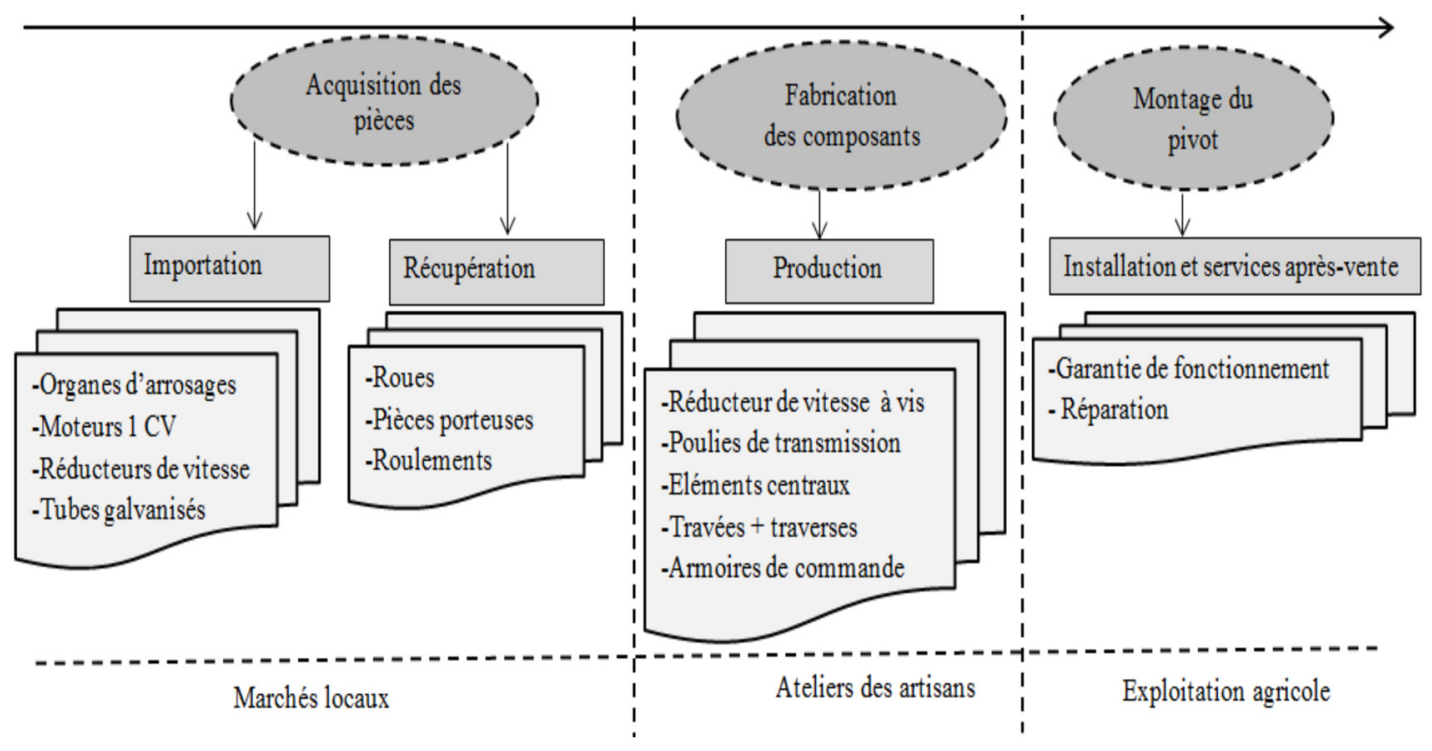

Fig. 4. Réseau de fourniture et prestation de service.

Fig. 4. Network of supply and provision of service.

poulies assurant la transmission entre le moteur et le réducteur de vitesse. Les fabricants-assembleurs, appelés localement « soudeurs », sont considérés comme des acteurs centraux qui sont en lien avec l'ensemble des intervenantsn: ils reçoivent les commandes des agriculteurs et se procurent les composants et matériaux nécessaires auprès des commerçants et artisans (tourneurs). Les ateliers des fabricants-assembleurs ressemblent à de véritables entreprises: ils engagent des ouvriers permanents et des saisonniers, ainsi que des stagiaires des centres de formation professionnelle. Les tâches de fabrication sont réparties selon un ordre précis : un ouvrier est chargé de l'ensemble des travées élémentaires de $12 \mathrm{~m}$, un second s'occupe des tourelles et des châssis et un autre se charge des éléments centraux.

Après finalisation de l'ensemble des parties composant le pivot, l'agriculteur est contacté pour les transporter jusqu'à sa parcelle. Les fabricants-assembleurs s'y déplacent ensuite pour le montage et la mise en route du pivot. Une garantie de fonctionnement couvrant une saison de production de pomme de terre est accordée aux agriculteurs, avec la prise en charge gratuite de la première réparation. Ces arrangements se font sur la base de la confiance et sans contrat écrit. La vente des pivots se fait parfois à crédit pour les agriculteurs n'ayant pas l'argent nécessaire pour payer comptant. Lorsque le recouvrement des créances tarde, le soudeur se trouve dans l'obligation d'acheter lui aussi les matériaux de fabrication à crédit.

Enfin, un électricien assure l'installation de l'armoire de commande électrique et son raccordement au moteur du pivot.

Aujourd'hui, la filière «pivot artisanal» constitue à elle seule une chaîne de production. Son évolution a permis le développement de petites entreprises de fabrication d'équipement d'irrigation, de réduire quelque peu le taux de chômage et de former sur le plan pratique des jeunes issus des centres de formation professionnelle. L'extension de cette filière offre aussi une opportunité de vente d'équipements aux importateurs et aux grossistes, ainsi que le recyclage des pièces vendues dans les garages et les casses automobiles.

\subsection{Diffusion du pivot et colonisation de nouveaux espaces agricoles}

La pomme de terre a été introduite au début des années 1990 par les services agricoles d'El Oued. Les agriculteurs y ont adhéré en raison des bons rendements régulièrement obtenus (30 à 40 tonnes par hectare). De plus, du fait de conditions climatiques clémentes en hiver, ils pouvaient produire en arrière-saison durant les mois de décembre et janvier, et vendre sur les marchés de gros sans concurrence avec les principaux bassins de production situés dans le Nord du pays (Benmihoub, 2015). Les agriculteurs ont commencé à irriguer par seguia, ou par aspersion classique, sur de faibles superficies. Au début des années 2000, l'apparition des pivots artisanaux en plus grand nombre a permis l'extension des superficies de pomme de terre, grâce à leur disponibilité, leur maniabilité et leur coût relativement abordable. Une surface d'environ un hectare peut être irriguée par un seul pivot artisanal dont le prix d'achat est de $1000 €$. En comparaison, l'équipement d'un hectare en goutte-àgoutte coûte environ $2000 €$ et un kit d'aspersion pour irriguer un hectare en couverture intégrale coûte entre $2000 €$ et $5000 €$, en fonction de la qualité de l'équipement choisi. Ces deux derniers systèmes exigent d'être enlevés lors du travail du sol et lors de la récolte, pour permettre l'accès des engins, contrairement au pivot artisanal qui peut être laissé sur place. La parfaite adéquation du système d'irrigation par pivot artisanal avec la production de la pomme de terre dans la région, s'est traduite par l'extension rapide des superficies cultivées.

Ce processus d'innovation et le développement de la culture de la pomme de terre ont attiré de nombreux investisseurs voulant pratiquer une agriculture entrepreneuriale fortement adossée aux marchés nationaux et très exigeante en capital financier. Les exploitations des investisseurs sont spécialisées dans la culture de pomme de terre, le bénéfice réalisé étant réinvesti dans l'extension des surfaces en 
Tableau 2. Caractéristique de l'échantillon des agriculteurs enquêtés.

Table 2. Characteristic of the sample of farmers interviewed.

\begin{tabular}{|c|c|c|c|c|c|c|}
\hline Types d'agriculteurs & \multicolumn{2}{|c|}{ Statut de l'agriculteur } & \multicolumn{3}{|c|}{ Mode de faire-valoir } & Nombre de pivots installés \\
\hline \multicolumn{7}{|l|}{ Nouveaux investisseurs } \\
\hline Petits investisseurs & 5 & 3 & 3 & 2 & 3 & $<10$ \\
\hline Fellah (cultivateurs) & 7 & / & 7 & / & / & $<10$ \\
\hline
\end{tabular}

pomme de terre. L'intérêt de l'association «pivot artisanal/ pomme de terre» réside notamment dans le fait que l'investisseur peut mettre en place progressivement plusieurs pivots pour ainsi étendre la superficie cultivée selon ses capacités d'investissement et le risque qu'il peut encourir. La plupart des producteurs de notre échantillon (17 sur 24) sont des investisseurs sans attache rurale directe dans le passé et issus d'autres secteurs économiques. Ils ont intégré le secteur de la production agricole après l'apparition du système pivot/ pomme de terre dans le Souf (Tab. 2). Nous distinguons deux types de nouveaux investisseurs:

- les grands investisseurs (9 agriculteurs sur 24 enquêtés) ont procédé à l'achat des terres, à la réalisation des puits et à l'installation de plus d'une dizaine de pivots, avec leurs propres moyens. Certains sont originaires d'El Oued mais n'y résident pas. Ils investissent une partie de leur capital pour couvrir les différents frais d'équipement et de fonctionnement de l'exploitation. Ils engagent des métayers qui vont gérer ces équipements et conduire les cultures. Ces métayers recrutent à leur tour un ou deux ouvriers permanents et des ouvriers journaliers, occasionnellement, durant le semis et la récolte. D'autres, résidant à El Oued, optent pour le mode de faire-valoir direct et emploient également un ou deux ouvriers permanents ainsi que des ouvriers journaliers pour les périodes des grands travaux;

- les petits investisseurs (8 agriculteurs sur 24 enquêtés), possèdent entre deux et six pivots. Ce sont surtout des jeunes producteurs. Ils ont moins de 35 ans et la plupart d'entre eux sont des fonctionnaires et des petits commerçants ayant des revenus limités. Par manque de capital foncier ou financier, ils louent des parcelles équipées en puits et pivots artisanaux et font appel à des associés pour l'acquisition des intrants et le paiement des frais de main-d'œuvre journalière et d'électricité. Ces jeunes contribuent ainsi, par la production de la culture de pomme de terre, à l'essor de l'agriculture locale aux côtés d'autres jeunes concernés par l'importation des pièces et d'équipements, leur assemblage et la commercialisation des pivots artisanaux.

À côté de ces investisseurs, sept agriculteurs de notre échantillon se considèrent comme fellahs, c'est à dire des agriculteurs anciennement installés. À l'inverse des nouveaux investisseurs, cette catégorie d'agriculteurs possède un plus petit nombre de pivots artisanaux ( 2 à 4 pivots) pour conduire un système maraîcher diversifié (pomme de terre, ail, oignon, arachide, ainsi que d'autres légumineuses). Ils sont proprié- taires et évitent la monoculture pour assurer le renouvellement de leur système agroécologique et préserver la fertilité du sol. La production est destinée à l'autoconsommation et au marché le bénéfice réalisé sera réinvesti dans un système maraîcher diversifié.

Cette nouvelle dynamique a permis non seulement de procurer des revenus aux populations locales par la création d'emplois dans les exploitations agricoles, mais a aussi encouragé le retour des grands entrepreneurs et commerçants originaires d'El Oued qui vivaient et menaient des activités non agricoles au Nord du pays. Cependant, cette dynamique est fondée sur l'utilisation d'une eau souterraine peu renouvelable, repose souvent sur des pratiques agricoles peu respectueuses de l'environnement et s'insère dans des marchés agricoles volatiles. En effet, la totalité des agriculteurs enquêtés accèdent à l'eau souterraine à travers des puits individuels ; généralement chaque puits permet d'alimenter un couple de pivots dont le fonctionnement se fait en alternance (jour et nuit). Le nombre de puits équipés en groupe motopompe est passé de 4200 en 1992 à 10600 en 2004 (Côte, 2006) et aurait fortement augmenté depuis selon les services agricoles. Certains agriculteurs pratiquent des rotations culturales; sur les 24 interviewés, 11 agriculteurs seulement cultivaient, en plus de la pomme terre, de l'arachide, de l'ail et de l'oignon. Les 13 autres agriculteurs ont opté pour la monoculture (pomme de terre en arrière-saison et en pleine saison). Cette dernière catégorie de producteurs se heurte généralement à des chutes rapides des rendements, pouvant atteindre $50 \%$ au bout de trois années ( 15 à 20 tonnes/ha au lieu des 30 à 40 tonnes/ha initiales). La monoculture de la pomme de terre est aussi une prise de risque considérable à cause de la volatilité des prix sur les marchés, qui constitue une forte source d'inquiétude pour tous les producteurs. Ainsi, les prix sur le marché national se sont-ils effondrés en 2015/2016 à cause d'un climat exceptionnellement doux dans les bassins de production au Nord, ce qui a conduit au ralentissement de la dynamique observée dans le Souf.

\section{Discussion et conclusion}

L'analyse de l'émergence puis de la conception et de la diffusion du pivot artisanal dans le Souf, par un réseau dense d'artisans et de commerçants en lien étroit avec des agriculteurs, a permis de comprendre le passage du modèle du grand pivot importé, promu par l'État, à un pivot artisanal peu coûteux et robuste, adapté aux choix culturaux des producteurs et aux conditions difficiles du Sahara. Cette évolution correspond au constat fait par Akrich et al. (1988) 
dans d'autres situations et de façon générique : "Adopter une innovation, c'est l'adapter; elle a besoin d'être transformée, modifiée en fonction du site où elle est mise en œuvre». Stewart (2007) considère les experts locaux comme un élément important dans le processus d'adaptation des nouvelles technologies du fait de leur influence sur, et des interactions au quotidien avec, les utilisateurs potentiels de l'innovation. Considérant que «la plupart des technologies impliquent la construction de répertoires collectifs et des référentiels de l'action et du sens » (Sørensen, 2006), nous pouvons postuler que le processus collectif d'innovation du pivot artisanal a produit de nouvelles normes et attentes, qui ont influencé à leur tour la manière dont la technologie est utilisée, ainsi que les pratiques des parties prenantes et les apprentissages nécessaires pour arriver à ce stade d'appropriation. Les artisans et les agriculteurs du Souf ont fait preuve d'une capacité de dialogue continue avec des référentiels multiples pour concevoir un dispositif d'irrigation de plus petite taille qui réponde aux objectifs des agriculteurs. Tout d'abord, des parcelles d'agriculteurs-utilisateurs ont servi de champ d'expérimentation pour les innovateurs pionniers pour résoudre progressivement les difficultés rencontrées par ces utilisateurs. Ces derniers ont ensuite démontré, par leur pratique, les performances du pivot artisanal pour la production de pommes de terre, avec deux saisons de pomme de terre par an et des rendements pouvant atteindre $40 \mathrm{t} / \mathrm{ha}$. Ceci a constitué une publicité grandeur nature attirant d'autres agriculteurs. Par la suite, et face à une demande croissante, ces artisans pionniers ont recruté et formé des ouvriers et des stagiaires des centres de formation. Ces artisans sont devenus, aujourd'hui, des fabricants connus dans la région que les agriculteurs consultent en cas de pannes. Les experts locaux sont aujourd'hui installés dans l'ensemble des zones de production, à proximité des utilisateurs avec lesquels ils continuent à interagir au quotidien.

Pour Sørensen (2006), l'adoption d'une technologie et les processus d'innovation centrés sur une question de production ou d'organisation interagissent avec leur environnement par un processus cognitif lié à l'apprentissage (Ameur et al., 2013). Dans notre cas, les artisans du Souf ont mis en œuvre un processus d'innovation incrémentiel à partir d'un pivot conventionnel importé où l'on a croisé savoirs et savoir-faire obtenus progressivement dans la pratique, et technologies et pièces détachées importées et récupérées chez les ferrailleurs. Cela a permis à ces experts locauxn - artisans et commerçants - de constituer, chemin faisant, une base partagée de connaissances. Ces connaissances concernent le fonctionnement du pivot, mais aussi le «marché potentiel» de ce pivot. En effet, ces experts locaux connaissent bien les systèmes de production, les attentes et le comportement des différents agriculteurs avec lesquels ils interagissent fréquemment. Ils adaptent continuellement la conception de l'équipement d'irrigation et sa distribution: déplacement et montage sur place dans les exploitations agricoles, production en série pour satisfaire la demande, ventes à crédit, service après-vente et réparation. Dans le cas étudié, une entrée par le processus d'innovation a permis de décrypter un réseau dense et complexe constitué de commerçants et artisans qui interviennent à différents niveaux: importation et distribution des pièces et équipements, fabrication et assemblage des composants, montage, installation et mise en route des pivots artisanaux et service après-vente. L'analyse des réseaux sociotechniques associée à un suivi des pratiques et des récits d'acteurs a permis de «rendre visibles des relations sociales invisibles » (Cross et al., 2002) en soulignant la complexité des liens sociaux et la diversité des dynamiques sociotechniques intervenant dans le processus d'innovation (Mahdi, 1993; Bouzidi et al., 2011).

Cet article a mis en évidence la reprise d'un modèle agrobusiness (céréales sous pivots conventionnels) par des communautés locales, en fabriquant un pivot artisanal et en l'utilisant sur une nouvelle culture. Selon Otmane et Kouzmine (2013), cette dynamique se caractérise par le «déploiement d'un système agricole technicisé et capitalistique visant la compétitivité et la rationalisation ». Ce système agricole productiviste, qui s'est mis en place et étendu rapidement, combine l'intensification à base d'intrants chimiques, la monoculture pour des marchés peu sécurisés, l'utilisation d'une eau souterraine peu renouvelable, et des arrangements sous forme de contrats agraires entre acteurs pour disposer d'un capital d'investissement et de trésorerie pour le fonctionnement des exploitations agricoles. De nombreuses questions restent en suspens, notamment l'impact de ce modèle de production sur les ressources naturelles (épuisement du sol et baisse des rendements, invasion de nématodes et diffusion de phytopathogènes, surexploitation et pollution des eaux souterraines), les stratégies développées par les agriculteurs pour faire face à la volatilité des marchés agricoles et le rôle de l'État pour accompagner et réguler ces nouvelles dynamiques agricoles. Ces questions et nos résultats doivent amener les structures de recherche et de développement à prendre en considération ces processus d'innovation pour envisager de les accompagner sans altérer les dynamiques locales et les relations de confiance entre les acteurs locaux, porteurs et utilisateurs de l'innovation. Cet accompagnement pourrait concerner l'amélioration des systèmes de production pour limiter les risques liés à une intensification monoculturale, l'utilisation plus rationnelle de l'eau ainsi que l'intégration de ces innovations locales dans les programmes de subvention. En outre, il est impératif de diversifier la production et de réfléchir de façon stratégique à l'ensemble de la filière depuis la production de semences et la récolte jusqu'au stockage, la transformation et la mise en marché, afin de mieux équilibrer la production et les marchés. Ceci permettra une meilleure attractivité pour ces régions et le retour des investisseurs qui en sont originaires vers l'activité agricole, pour participer à la satisfaction alimentaire et à la diversification des sources de revenus du pays.

\section{Références}

Akrich M, Callon M, Latour B. 1988. À quoi tient le succès des innovations? 1: l'art de l'intéressement; 2 : le choix des porteparole. Gérer et Comprendre. Annales des Mines 11-12: 4-17.

Ameur F, Hamamouche MF, Kuper M, Benouniche M. 2013. La domestication d'une innovation technique: la diffusion de l'irrigation au goutte-à-goutte dans deux douars au Maroc. Cah Agric 22: 311-318. doi: 10.1684/agr.2013.0644.

Benmihoub A. 2015. Modélisation du comportement des agriculteurs en situation de risque sur le revenu. Cas des producteurs de la pomme de terre. Thèse de doctorat en sciences agronomiques, École nationale supérieure agronomique, Alger, 157 p. 
Benouniche M, Kuper M, Poncet J, Hartani T, Hammani A. 2014. Quand les petites exploitations adoptent le goutte-à-goutte: initiatives locales et programmes étatiques. Cah Agric 20: 40-7. doi: 10.1684/agr.2011.0476.

Benouniche M, Errahj M, Kuper M. 2016. The seductive power of an innovation: enrolling non-conventional actors in a drip Irrigation community in Morocco. Journal of Agricultural Education and Extension 22(1): 61-79. doi: 10.1080/ 1389224x.2014.977307.

Bisson J. 2003. Mythes et réalités d'un désert convoité, le Sahara. Paris : le Harmattan. 480 p.

Bouzidi Z, Abdellaoui EH, Faysse N, Billaud JP, Kuper M, Errahj M. 2011. Dévoiler les réseaux locaux d'innovation dans les grands périmètres irrigués. Cah Agric 20: 34-9. doi: 10.1684/agr.2011.0471.

Côte M. 2006. Si le Souf m'était conté. Constantine : Édition Média plus. 135 p.

Cross R, Borgatti SP, Parker A. 2002. Making invisible work visible: using social network analysis to support strategic collaboration. Calif Manage Rev 44: 25-46.

Dubost D, Larbi Y. 1998. Mutations agricoles dans les oasis algériennes : l'exemple des Ziban. Sécheresse 9(2): 103-10.

Edquist C. 2005. Systems of innovation. In: Fagerberg J,Mowery DC, Nelson RR, eds. The Oxford handbook of innovation. New York: Oxford University Press. pp. 181-208.
Institut national d'irrigation et drainage (INSID). 2011. Carte d'occupation des sols d'Algérie.

Knickel, K, Brunori G, Rand S, Proost J. 2009. Towards a better conceptual framework for innovation processes in agriculture and rural development: from linear models to systemic approaches. Journal of Agricultural Education and Extension 15(2): 131-146.

Mahdi M. 1993. Innovation et système social. Options Méditerranéennes 2: 17-30.

Office national de la météorologie (ONM). 2012. Bulletins mensuels de relevés des paramètres climatologiques en Algérie de 1992 à 2012. Centre climatologique national (CCN), Dar El Beïda, Alger.

Ortiz O, Orrego R, Pradel W, Gildemacher P, Castillo R, Otiniano R, et al. 2013. Insights into potato innovation systems in Bolivia, Ethiopia, Peru and Uganda. Agricultural Systems 114: 73-83.

Otmane T, Kouzmine Y. 2013. Bilan spatialisé de la mise en valeur agricole au Sahara algérien. Cybergeo: European Journal of Geography. Espace, Société, Territoire 632. doi: 10.4000/ cybergeo.25732. Disponible sur http://cybergeo.revues.org/25732.

Sørensen KH. 2006. Domestication: the enactment of technology. In: Berker T, Hartmann M, Punie Y, Ward KJ, eds. Domestication of Media and Technology. London: Open University Press.

Stewart J. 2007. Local experts in the domestication of information and communication technologies. Information, Communication \& Society 10: 547-69.

Citation de l'article : Ould Rebai A, Hartani T, Chabaca MN, Kuper M. 2017. Une innovation incrémentielle : la conception et la diffusion d'un pivot d'irrigation artisanal dans le Souf (Sahara algérien). Cah. Agric. 26: 35005. 